\title{
A Torque Discontinuity Free New Hybrid PWM Approach for High Speed Induction Motor Drives
}

\author{
Habibur Rehman, Roufin Mahmood, and Taimoor Shah \\ Department of Electrical Engineering, School of Engineering, American University of Sharjah, P.O. Box 26666, Sharjah, UAE \\ Correspondence should be addressed to Roufin Mahmood; b00035111@alumni.aus.edu
}

Received 4 September 2013; Revised 26 January 2014; Accepted 10 February 2014; Published 2 April 2014

Academic Editor: Don Mahinda Vilathgamuwa

Copyright ( 2014 Habibur Rehman et al. This is an open access article distributed under the Creative Commons Attribution License, which permits unrestricted use, distribution, and reproduction in any medium, provided the original work is properly cited.

\begin{abstract}
This paper designs a hybrid speed controller in which a Sine Triangle Pulse Width Modulated (SPWM) inverter is used below the base speed and a square wave inverter is employed above the base speed. The two inversion techniques complement each other for their advantages and disadvantages below and above the base speed. This paper proposes a unique strategy for the transition between SPWM and square wave by simply setting the frequency of the carrier signal equal to zero. The proposed methodology in a way uses only one inversion technique and realizes a seamless transition from the SPWM to square wave compared to conventional method in which modes are simply switched from SPWM to square wave and vice versa when the speed changes above and below the base speed, respectively. Computer simulations show that the proposed technique has smoother torque transition and thus a better speed response compared to conventional approach of inverter mode switching around the base speed. The performance of proposed hybrid approach is also validated on a small prototype induction motor through experimental results.
\end{abstract}

\section{Introduction}

The high speed, medium to high power induction motor drives have a wide variety of applications like traction drive of high speed railway, alternative energy vehicles, and many other industrial applications. However, in these high speeds, high power drives, the sine triangle PWM technique becomes impractical because of lower DC bus utilization, higher fundamental frequency at which the motor operates during high speed operation, and unavailability of high power devices with high switching frequencies. Thus, for all such applications, square wave inverters are being employed [15]. However, the square wave inverter suffers from the harmonics problems which are currently being controlled using adjustable filters for such drive systems [6, 7]. The harmonics problem becomes worse as motor speed becomes lower than the base speed. The low frequency operation reduces the motor leakage inductance which increases the harmonics current and thus reduces the motor efficiency badly [2] and also causes torque pulsations in the machine. Another problem at the low speed operation is that motor voltage needs to be proportionally adjusted to the motor speed. This voltage for a fixed DC bus voltage cannot be controlled using a square wave inverter, while with a Sine Triangle Pulse Width Modulated (SPWM) inverter the inverter output voltage can be controlled by controlling the amplitude of reference signal. To overcome these problems, this paper designs a hybrid PWM technique in which a SPWM inverter is used below the base speed and a square wave inverter is used above the base speed. The proposed strategy is implemented for volts per hertz control of induction machine. The overall drive system is shown in Figure 1. The transition from the sine triangle to square wave inverter above the base speed is realized in a new and unique way by simply setting the frequency of the carrier signal equal to zero. Thus, the proposed method uses only SPWM technique and yet realizes the SPWM operation below the base speed and square wave operation above the base speed. This avoids the torque discontinuity which normally occurs while switching between the sine triangle and any other PWM technique to square wave $[8,9]$. The proposed strategy is implemented on a small low power, low voltage prototype motor in the lab. A small motor is used just to prove the concept but the targeted applications are medium to high power and high speed drives where high power 


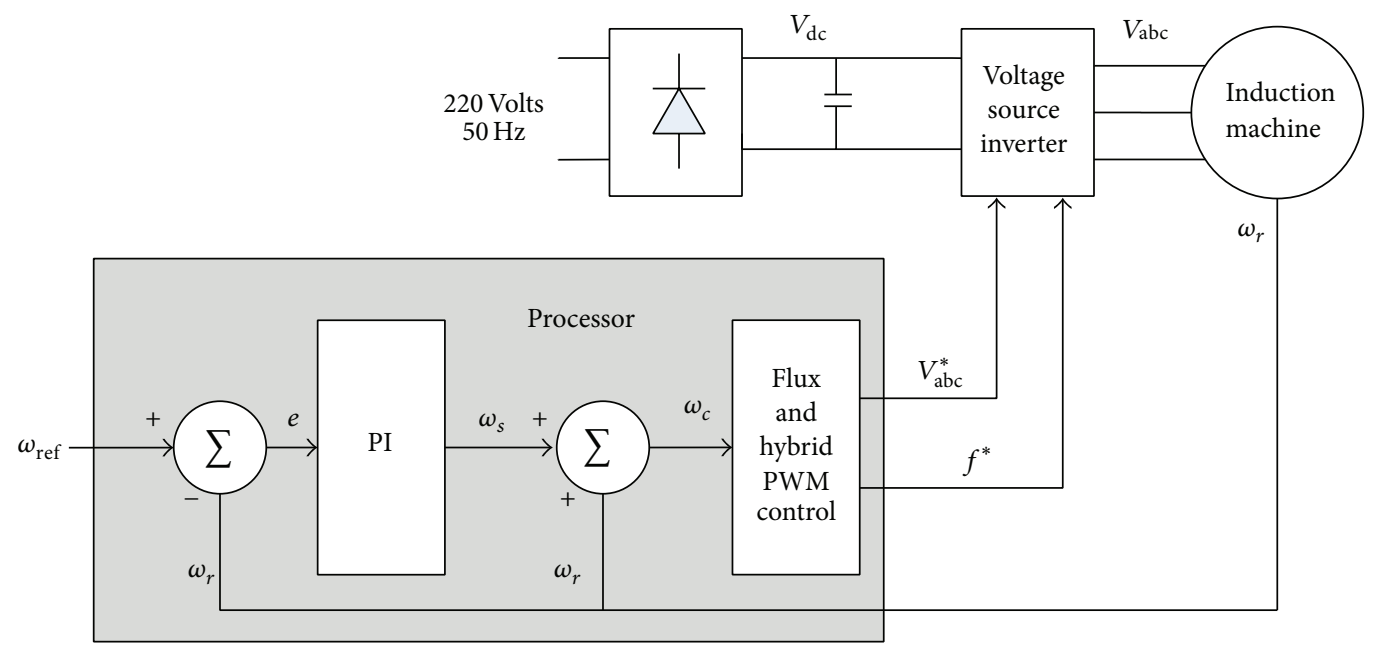

FIGURE 1: Overall speed control system.

and high frequency switching devices are not yet available. Therefore, the carrier frequency in this work is set to a relatively lower value of $1050 \mathrm{~Hz}$ below the base speed. The SPWM and square inverters are compared and proposed strategy is elaborated in Section 2; Section 3 presents the simulation results comparing the conventional approach of mode switching and the proposed technique, and finally experimental results are included in Section 4 and concluding remarks are made in Section 5 of this paper.

\section{The Proposed Hybrid Approach}

The square wave and sine triangle inverter are the two widely used techniques for the speed control of the induction machine. The harmonics analysis of a square wave inverter yields the following [10]:

$$
V_{L-L}=\sum_{n=1,3,5, \ldots}^{\infty} \frac{4 V_{s}}{n \pi} \sin \left(\frac{n \pi}{2}\right) \sin \left(\frac{n \pi}{3}\right) \sin \left(\omega t+\frac{\pi}{6}\right) .
$$

Equation (1) indicates that 5th and 7th harmonics are the most dominant ones for a square wave inverter. Thus, at the low speed operation with, for example, a reference frequency of $5 \mathrm{~Hz}$, the first two dominant harmonics occur at the frequencies of $25 \mathrm{~Hz}$ and $35 \mathrm{~Hz}$. These harmonics are present at frequencies very close to the fundamental component and hence cannot be filtered out. At such low frequency operation the leakage inductance of the machine will be very small and its effect in the current cannot be filtered out which even affects the machine torque badly and causes torque pulsations. Another major drawback of the square wave inverter operation at the low speed is that only voltage frequency can be controlled and not the magnitude. The SPWM inverter on the other hand can adjust both the voltage and frequency required by the scalar control of the machine. The harmonic profile of the sine triangle PWM inverter is given by [10]

$$
f_{h}=\left(j m_{f} \pm k\right) f_{1}
$$

where $j=1,3,5, \ldots$ for $k=2,4,6, \ldots$ and $j=2,4, \ldots$ for $k=1,5,7, \ldots$, such that $j m_{f} \pm k$ is not a multiple of three. Equation (2) indicates that, for a sine triangle inverter, the lowest harmonic present in the output voltage is shifted to the switching frequency of the inverter. The harmonics in the inverter output voltage waveform appear as sidebands, centered on the switching frequencies and their multiples, that is, around $m_{f}, 2 m_{f}, 3 m_{f}$, and so on. Thus, the harmonics for a sine triangle inverter are shifted to higher frequencies which are easy to filter and also their effect due to high frequency is not dominant in the torque pulsations especially at low speed. The SPWM inverter adjusts both the voltage and frequency required for the scalar control technique. However, the problem with SPWM is its poor DC bus utilization above base speed where a maximum voltage is desired. The square wave inverter on the other hand has an advantage of a better DC bus utilization which can be used to apply maximum possible voltage to the motor. Therefore, a square wave inverter becomes a better choice at high speed as compared to the SPWM inverter. The DC bus utilization is second major advantage of the proposed hybrid technique. The rms fundamental line voltage for a square wave inverter is given by [10]

$$
V_{L 1}=\frac{4 V_{\mathrm{dc}} \sin 60^{\circ}}{\sqrt{2} \pi}=0.7797 V_{\mathrm{dc}} .
$$

As for SPWM (for $m_{a} \leq 1.0$ ), the fundamental frequency component in the output voltage varies linearly with the amplitude modulation ratio $m_{a}$ and can be represented as

$$
V_{L 1}=0.612 m_{a} V_{\mathrm{dc}} \text {. }
$$

The maximum possible rms value of the fundamental component with SPWM, set at a modulation index of 1 , is $0.612 V_{\mathrm{dc}}$, which is approximately $21.5 \%$ less than that of the square wave inverter. This problem of poor DC bus utilization becomes more critical when the motor has to be operated at higher speeds. The other problem with SPWM operation at high speeds is that the fundamental frequency may reach about 


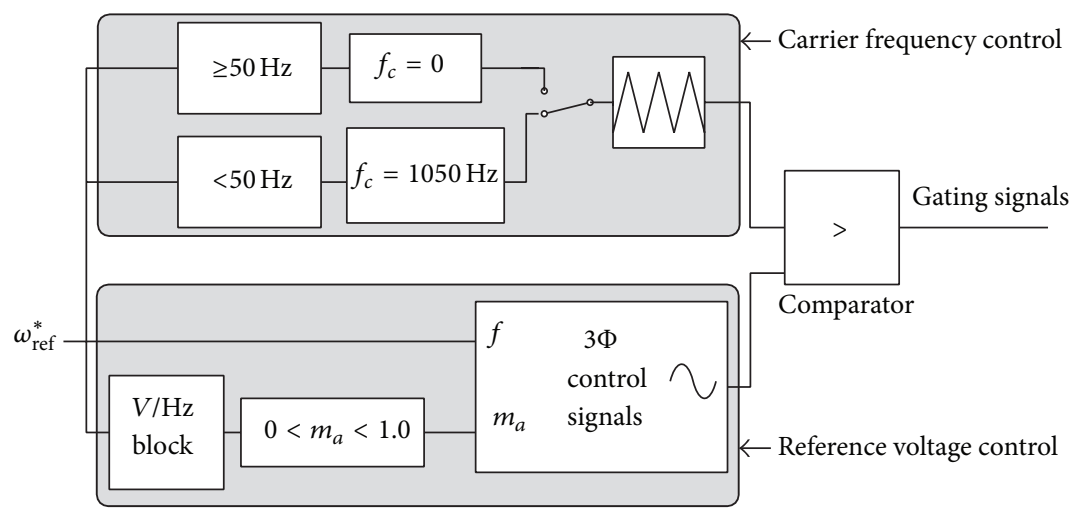

FIGURE 2: Switching between SPWM and square wave techniques.

$1 \mathrm{kHz}$ which requires very high switching frequency devices whose availability with high power ratings is an issue. Thus, the solution is to use a SPWM at low speeds and a square wave inverter at high speed operation.

Based on the comparison of the square wave and SPWM inverter outlined above, the square wave and SPWM techniques complement each other in the two regions of operation, that is, below and above the base speed. The SPWM has higher frequency harmonics around the switching frequency below the base speed, while a square wave inverter has better DC bus utilization and inverter switches are to relatively operate at lower switching frequency for high speed operation. Thus, this paper proposes the motor controller design which uses SPWM below the base speed and square wave inverter above the base speed. In order to realize the square wave inverter, the carrier frequency of the triangular waveform is set to zero which makes seamless transition from the SPWM to a square wave inverter. The output voltage does not remain a train of pulses and is held constant at a value of $0.78 \mathrm{~V}_{\mathrm{dc}}$ and thus the inverter essentially becomes a square wave inverter. The square wave inverter supplies a higher line-to-line voltage to the motor and thus higher DC bus utilization can be achieved.

Figure 2 details the methodology for proposed hybrid operation and gating signal generation. It consists of two blocks; one for the carrier frequency control and the other for controlling the reference voltage. The frequency control block is responsible for generating the triangular carrier waveform with a frequency, $f_{c}$, depending on the command speed whether it is below or above the base speed. The carrier frequency is set to $1050 \mathrm{~Hz}$ if command speed is below the base speed and is set to zero if the reference speed is equal or above the base speed. The reference voltage control block is used to generate the 3-phase reference signals with a given modulation index, $m_{a}$, and frequency. If the desired speed is below base speed, the $\mathrm{V} / \mathrm{Hz}$ block determines a voltage level and, consequently, a value for $m_{a}$, to maintain the constant flux ratio. If it is above base speed, $m_{a}$ is kept constant. The 3 -phase reference signals are then compared with the carrier and the gating signals are generated.
TABLE 1: Prototype induction motor parameters.

\begin{tabular}{lcc}
\hline $415 \mathrm{~V}$ & $0.4 \mathrm{~A}$ & $175 \mathrm{~W}$ \\
\hline$L_{\mathrm{ls}}=L_{\mathrm{lr}}=87 \mathrm{mH}$ & $L_{m}=1.9 \mathrm{H}$ & $1475 \mathrm{rpm}$ \\
$R_{s}=50 \Omega$ & $R_{r}=16 \Omega$ & 4 poles \\
\hline
\end{tabular}

\section{Simulation Results}

The proposed system is simulated using MATLAB/Simulink to validate its performance. The parameters of the $175 \mathrm{~W}$ prototype motor used for the simulations and experimentation are given in Table 1. In the simulations as well as the experiments, $f_{c}$ is chosen to be $1050 \mathrm{~Hz}$ to eliminate the triplen harmonics for SPWM operation. The algorithm is validated on a low power prototype motor but the targeted applications are medium to high power drive systems. Thus, the carrier frequency chosen is low because, for high power high speed applications, high frequency switching devices are not available.

The first set of simulation compares the operations of square wave and SPWM inverter below and above the base speed and the results for the voltage and current waveforms are shown in Figures 3-5. The reference speed below the base speed is set at $750 \mathrm{rpm}$ and that of above base speed is set at $1800 \mathrm{rpm}$. Figure 3 shows the voltage waveform at $750 \mathrm{rpm}$. Since this is below base speed, the voltage at the DC bus, $V_{\mathrm{dc}}$, is manually adjusted at $300 \mathrm{~V}$ to maintain the rated flux for the $V / f$ operation at this speed. While using the SPWM inverter, the DC bus is retained at its full capacity, that is, 535 V. The FFT analysis is shown in Figures 3(b) and 3(d) for the square wave and SPWM, respectively. It can be seen that the square wave inverter has presence of lower order harmonics in contrast to SPWM, with the former having its first two harmonics at $125 \mathrm{~Hz}$ and $175 \mathrm{~Hz}$, that is, the 5th and 7th harmonics. The SPWM FFT shows that the first two harmonics occur at $1000 \mathrm{~Hz}$ and $1100 \mathrm{~Hz}$, centered around the carrier frequency at $f_{c} \pm 2 \cdot f_{1}$, where $f_{1}$ is the fundamental frequency. Using the square wave inverter the voltage at the fundamental frequency is $165 \mathrm{~V}$ whereas in SPWM it is found to be $147 \mathrm{~V}$. This is in accordance with the previous discussion 


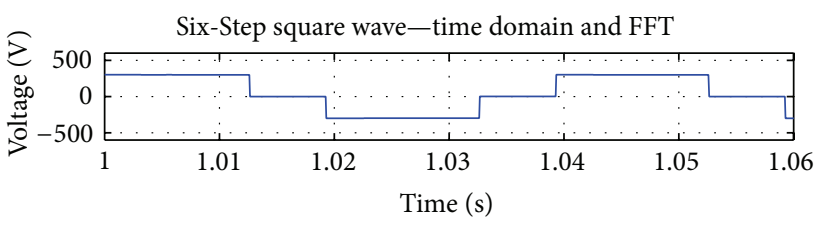

(a)

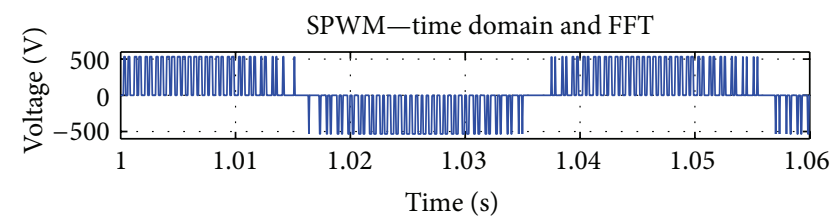

(c)

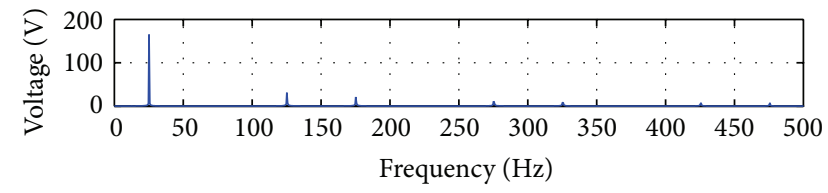

(b)

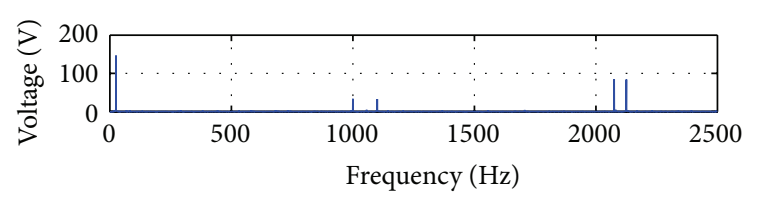

(d)

FIGURE 3: Voltage profile for $750 \mathrm{rpm}$.

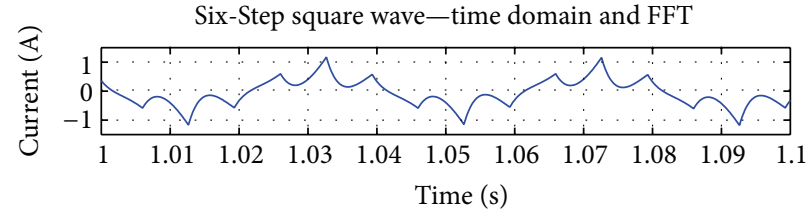

(a)

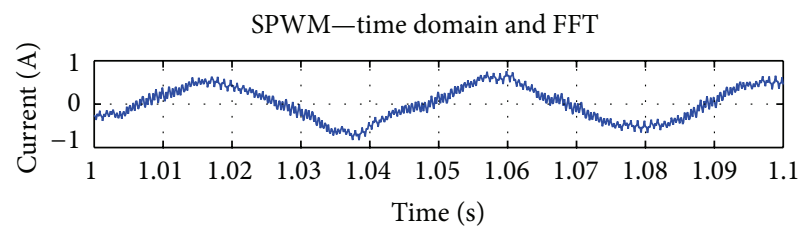

(c)

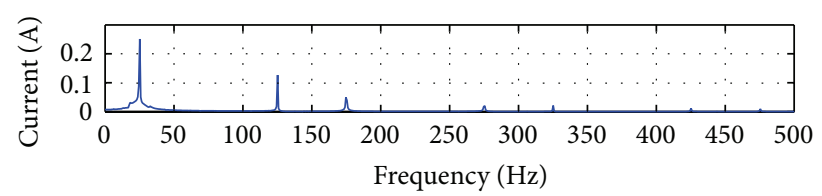

(b)

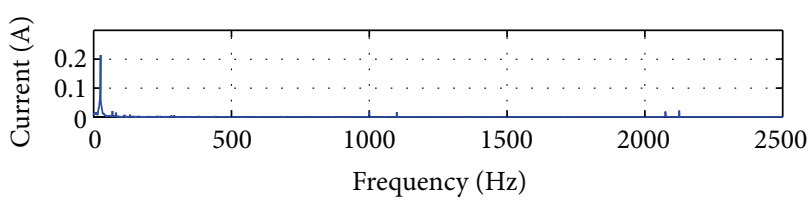

(d)

FIGURE 4: Current profile for $750 \mathrm{rpm}$.

of a higher line-to-line voltage in the square wave inverter. However, this advantage cannot be put to good effect in closed loop operation below base speed since there is an issue with maintaining the constant flux ratio.

Figures 4(a) and 4(c) show the time domain currents for the $750 \mathrm{rpm}$ operation. Figures 4(b) and 4(d) show the current harmonics appearing at the same frequencies as those of the voltage harmonics. However, their magnitudes are lower in relation to the fundamental component since the load is an induction motor. It should be noted that current waveform of the SPWM inverter is closer to a sine wave as compared to the current waveform of the square wave inverter. This is because the motor's inductive behavior prevents rapid changes in current as the inverter gates are rapidly switched on and off. The most dominant harmonic for a square wave inverter is the 5 th one occurring at about $125 \mathrm{~Hz}$ and its magnitude is quite high which can lower the machine efficiency and cause torque ripples in the machine. The SPWM inverter's most dominant harmonic is around the switching frequency of $1050 \mathrm{~Hz}$ and its magnitude is quite low thus favoring the motor operation using SPWM at low speed.

Figure 5 shows the voltage profile at a reference speed of $1800 \mathrm{rpm}$ for both types of inverters. The time domain signals are shown in Figures 5(a) and 5(c) and the FFT is shown in Figures 5(b) and 5(d). In both tests the DC bus is maintained at its full capacity. During operation above base speed, the voltage and current harmonics of the six step show the same behavior, that is, more pronounced (high magnitudes) lower order harmonics. The first two harmonics appear at $300 \mathrm{~Hz}$ and $420 \mathrm{~Hz}$ as can be seen in Figure 5(b). The lower order harmonics in this case do not pose that big of a disadvantage, since their magnitude is low and they do not appear close to the fundamental component. The magnitude of fundamental voltage for the square wave and SPWM mode is found to be $293 \mathrm{~V}$ and $178 \mathrm{~V}$, respectively. It is evident in this case that the square wave inverter is clearly more desirable since it yields a higher line-to-line voltage which is very much needed for the high speed operation. Hence, it can be concluded that above the base speed square wave technique has the advantage of better DC bus utilization applying a higher lineto-line voltage to the machine as illustrated by (3). Also, in contrast to the low speed operation there is no need to adjust the DC voltage because it is set at its full value.

The second set of computer simulations is performed to investigate the performance of proposed technique for the speed control response and torque ripples. Two cases are 


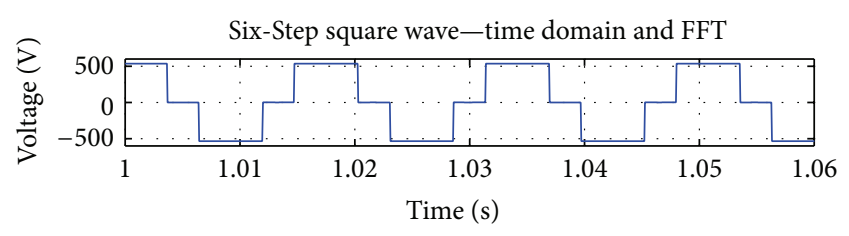

(a)

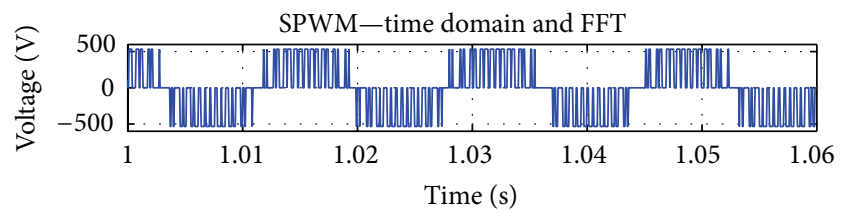

(c)

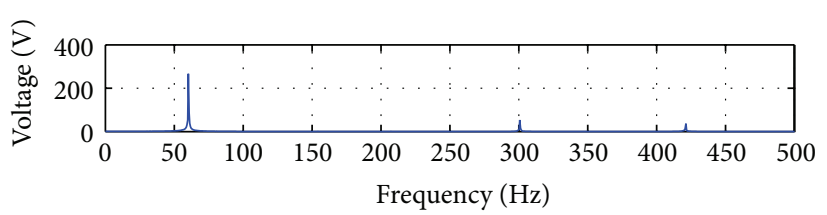

(b)

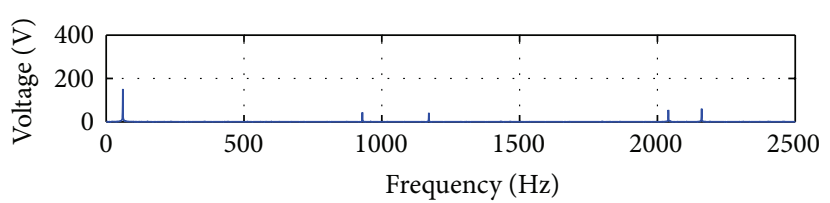

(d)

FiguRE 5: Voltage profile for $1800 \mathrm{rpm}$.

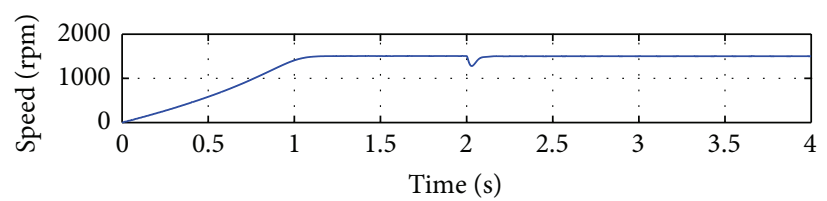

(a)

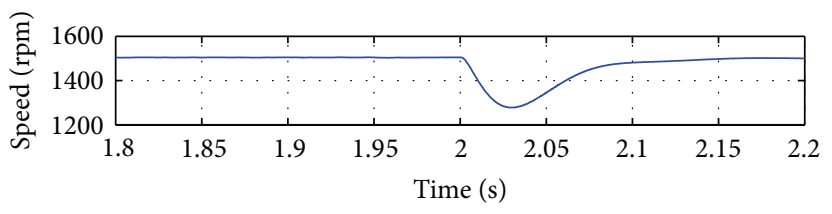

(c)

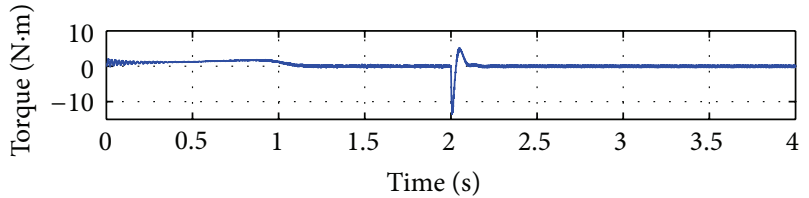

(b)

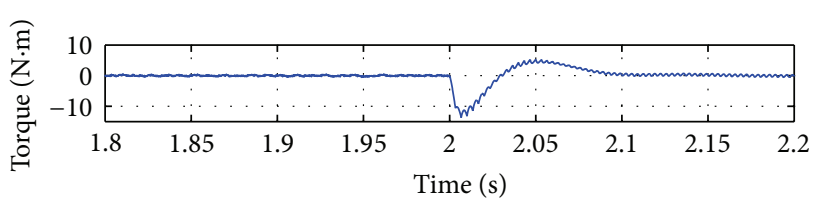

(d)

Figure 6: Case I: steady-state speed and torque response with mode switching.

presented here: Case I, in which the inverter is switched from sine triangle to square wave inverter and Case II that realizes the suggested mode switching from sine triangle to square wave by suppressing the $f_{c}$ to zero. These cases are tested for both the steady-state and the dynamic speed operation.

In the steady-state operation, the speed is kept at $1500 \mathrm{rpm}$ and the mode is changed using the technique mentioned in Case I. The plots obtained are shown in Figure 6. Figures 6(a) and 6(b) are the speed and the torque plots, respectively, while Figures 6(c) and 6(d) are the zoomed plots at the transition. It can be seen that the speed drops from $1500 \mathrm{rpm}$ to $1280 \mathrm{rpm}$ before it rises to its steady-state value of $1500 \mathrm{rpm}$. Consequently, for this speed drop there is a negative torque with a magnitude of $13.43 \mathrm{~N} \cdot \mathrm{m}$ before it stabilizes to a steadystate value. To avoid this high torque discontinuity, it is proposed to use the method of Case II, whose results are presented in Figure 7. Figure 7 shows that, in this case, although the speed varies during the transient, it does not exceed a value more than $20 \mathrm{rpm}$ from its steady-state value. The torque transient is also improved as it varies only between 2.4 and $-2 \mathrm{~N} \cdot \mathrm{m}$ during this transition.

Next, the two cases are investigated for their performance while the command speed changes from just below the base speed to above base speed. Test speeds of $1450 \mathrm{rpm}$ and
$1600 \mathrm{rpm}$ are used to prove the case in point. Figures 8 and 9 depict the results of the test scenarios for Cases I and II, respectively, where the speed waveforms are shown in subplots in Figures 8(a) and 9(a) and torque waveforms are shown in Figures 8(b) and 9(b). In Case I, it can be seen from the enlarged plots in Figures $8(\mathrm{c})$ and $8(\mathrm{~d})$ that the torque transition when the command speed changes from $1450 \mathrm{rpm}$ to $1600 \mathrm{rpm}$ is quite significant. The torque changes to a negative value of $11.4 \mathrm{~N} \cdot \mathrm{m}$ which initially drops the machine speed to $1360 \mathrm{rpm}$ before it rises to $1600 \mathrm{rpm}$. On the contrary, the torque discontinuity which is quite evident in Case I does not occur in Case II. A positive torque of $2.6 \mathrm{~N} \cdot \mathrm{m}$ is generated to increase the motor speed and the motor speed smoothly settles to the desired value. The speed does not drop during this transition in contrast to Case I in which speed dips to $1360 \mathrm{rpm}$ before changing to the desired reference. This shows that the dynamic performance with the proposed algorithm has very much improved and provides a smoother speed transition proving that the proposed method of mode switching is a better alternative.

Finally, Figure 10 shows the simulation results from changing the reference speed from $1200 \mathrm{rpm}$ to $1800 \mathrm{rpm}$ and consequently to $2200 \mathrm{rpm}$ with the proposed algorithm. Figure 10(b) shows how the voltage waveforms change from 


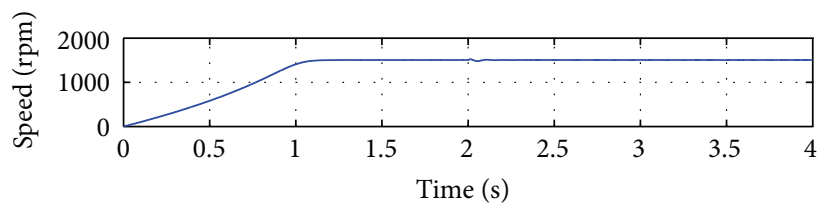

(a)

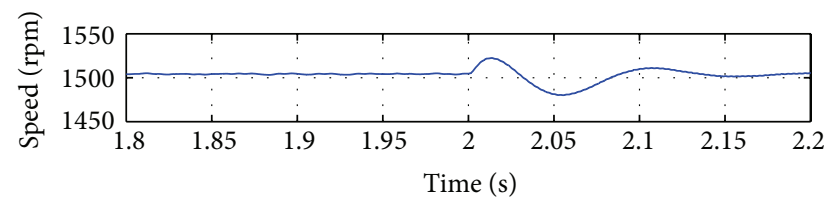

(c)

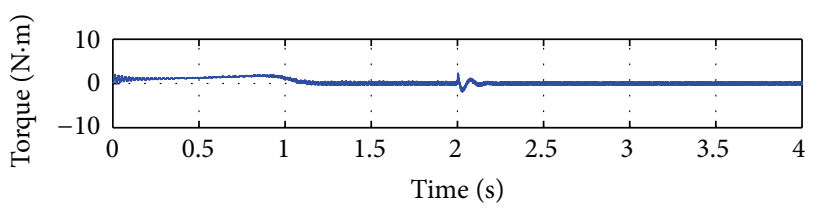

(b)

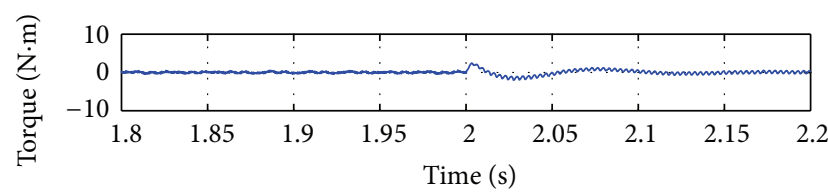

(d)

FIGURE 7: Case II: steady-state speed and torque response with proposed algorithm.

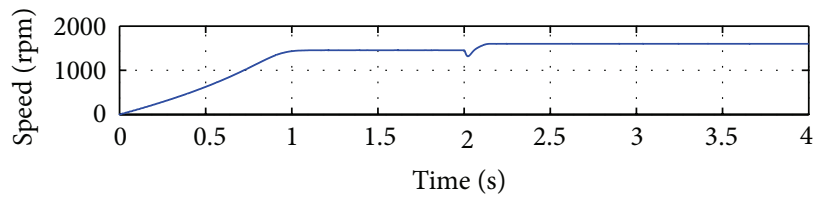

(a)

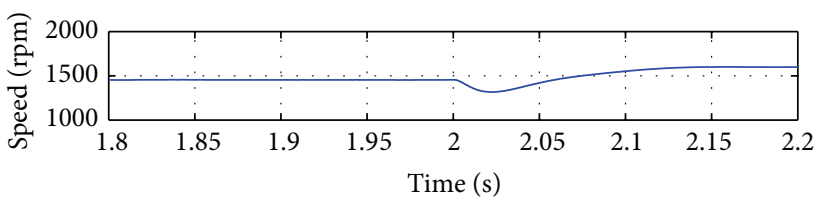

(c)

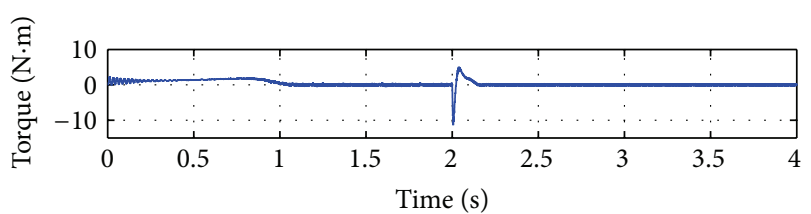

(b)

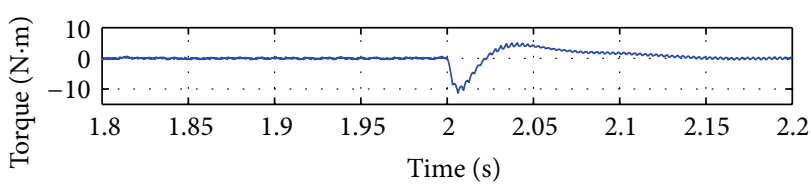

(d)

FIGURE 8: Case I: dynamic speed and torque response with mode switching.

a PWM waveform to that of a square wave when command speed changes to a speed above base speed. The current waveform is also shown in Figure 10(c). Thus, the intensive computer simulations performed above validate the proposed algorithm and prove that it has lesser torque ripples and a better speed response when the inverter mode is changed from PWM to a square wave mode of operation for high speed control of induction machine.

\section{Experimental Results}

In order to validate the simulation results, experiments are performed on a 415 Volt, 175 Watt prototype $50 \mathrm{~Hz}, 4$ pole, and 3- $\Phi$ induction machine whose rated speed is about $1475 \mathrm{rpm}$. The motor parameters are already included in Table 1 . The dSPACE CP1104 board is used for implementing the control algorithms. The experimental setup on which the tests are carried out is shown in Figure 11. Tests are carried out with a carrier frequency of $1050 \mathrm{~Hz}$. Figure 12 represents the voltage profile for the case below base speed, that is, $750 \mathrm{rpm}$. Figures 12(a) and 12(b) represent the line voltage waveform using square wave inverter and the harmonics profile, respectively. Similarly, Figures 12(c) and 12(d) represent the line voltage using sine triangle modulation and the corresponding harmonics analysis. The experimental results like the simulations show that the harmonics in the square wave occur at lower frequencies in comparison to the SPWM where they are located at higher frequencies. Hence, the harmonics of sine triangle inverter can be filtered out easily as compared to the square wave inverter. This advantage of sine triangle inverter is one major reason for using this modulation technique for below base speed operations.

Figure 13 shows the experimental results for a wide range of speed control. The motor is initially operated below the base speed at $1200 \mathrm{rpm}$. The command speed is stepped up to $1800 \mathrm{rpm}$ and finally to $2200 \mathrm{rpm}$ well above the base speed. The system operates using sine triangle modulation below base speed conditions, that is, below $1475 \mathrm{rpm}$, while it utilizes the square wave inverter above the base speed (Figure 14(b)). It can be seen that the system successfully adjusts the actual speed of the motor very close to the reference speed for both types of modulation techniques. The system also successfully switches from sine triangle to square wave without any discontinuity in the speed response. Figure 13(b) shows the voltage waveform for the complete operation of the machine at the three different speeds. Figure 13(c) shows the machine 


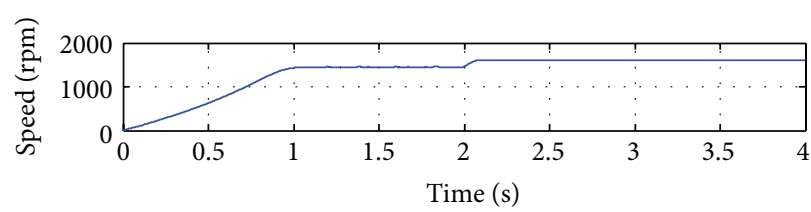

(a)

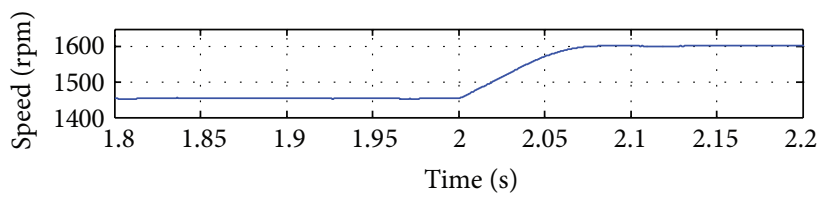

(c)

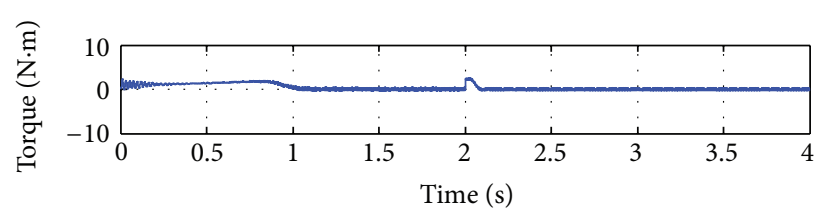

(b)

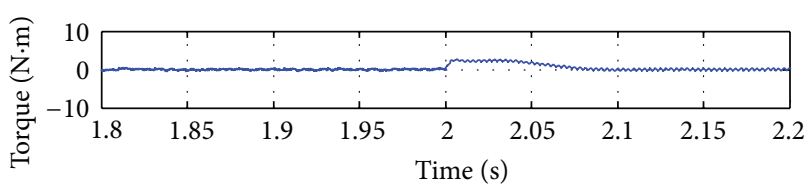

(d)

FIGURE 9: Case II: dynamic speed and torque response with proposed algorithm.

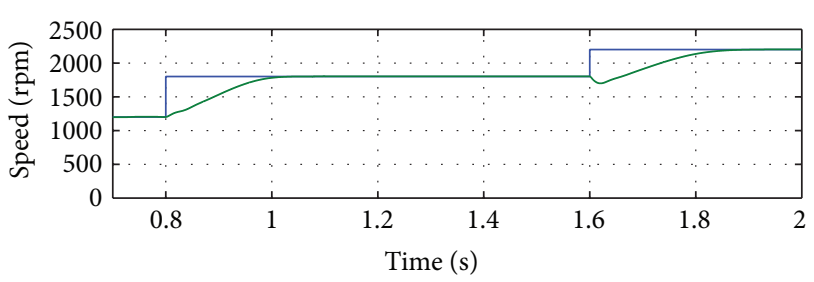

(a)

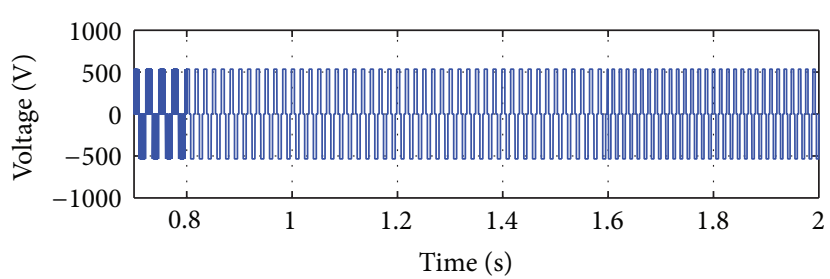

(b)

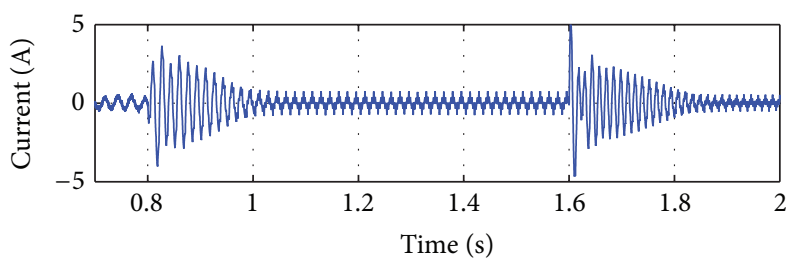

(c)

FIGURE 10: Simulation results for below and above base speed with the proposed algorithm.

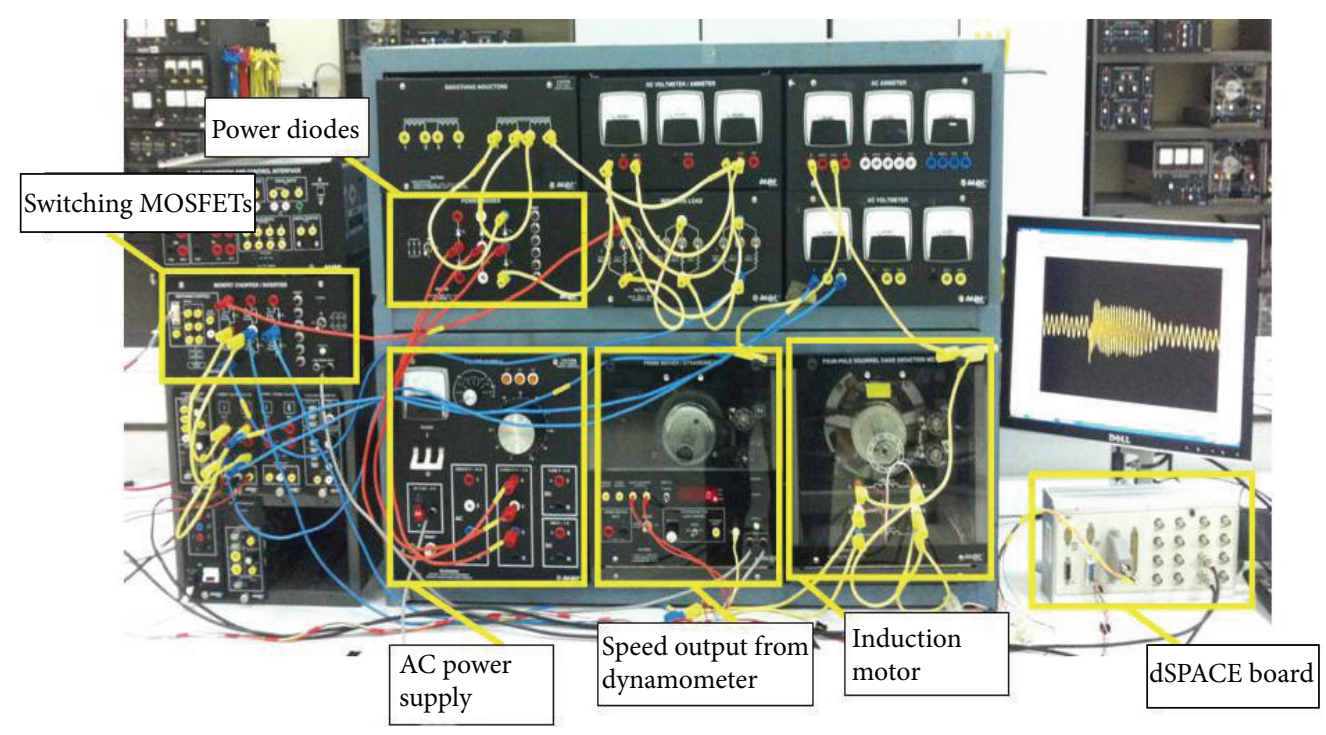

FIGURE 11: Experimental platform. 


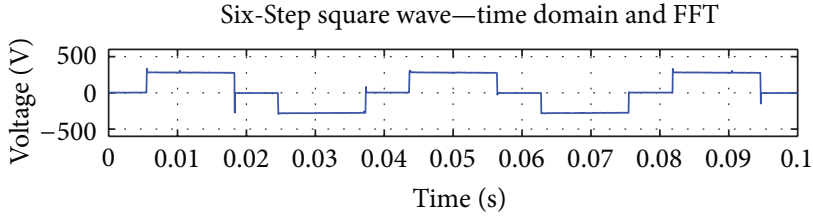

(a)

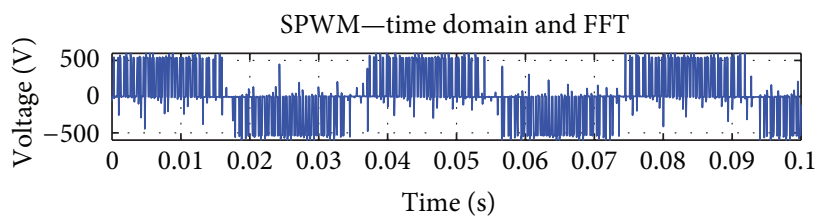

(c)

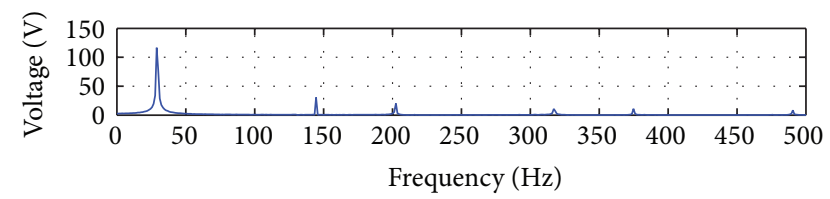

(b)

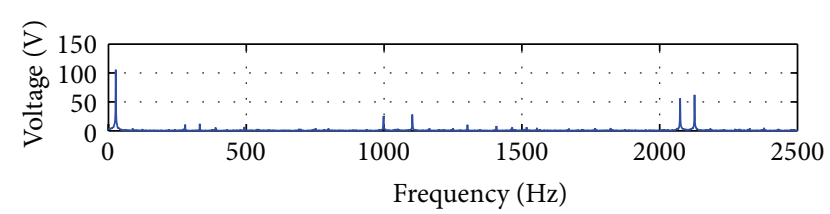

(d)

FIGURE 12: Voltage profile for $750 \mathrm{rpm}$.

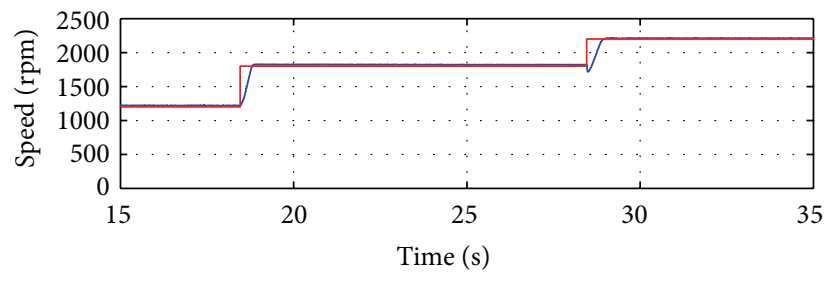

(a)

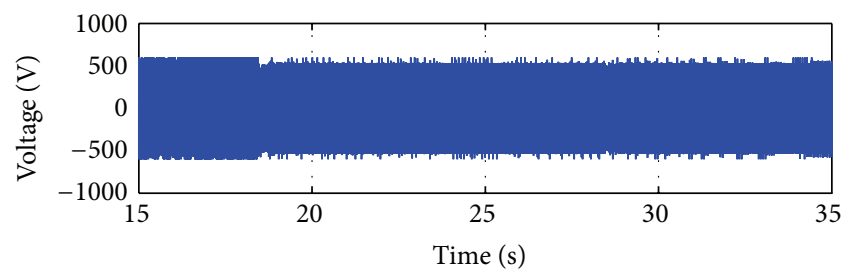

(b)

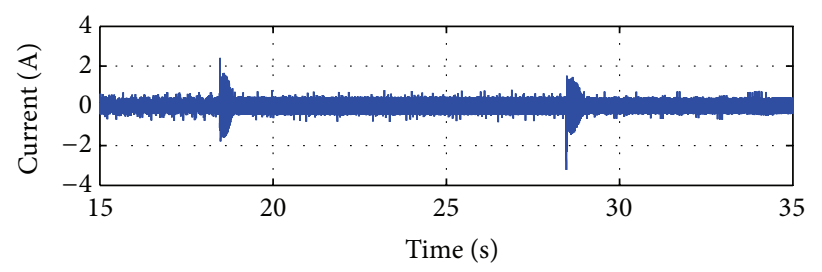

(c)

FIGURE 13: Experimental results for below and above base speed operation.

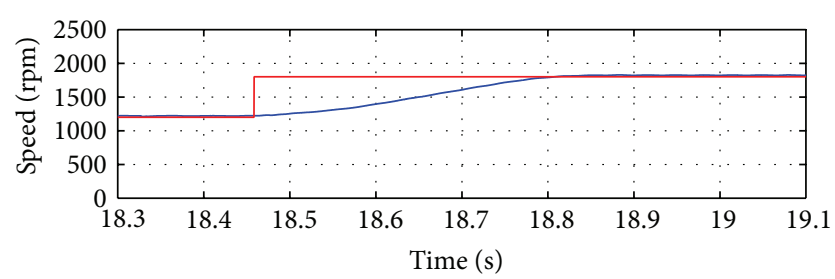

(a)

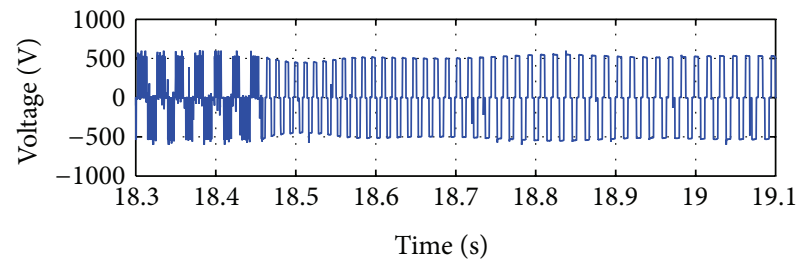

(b)

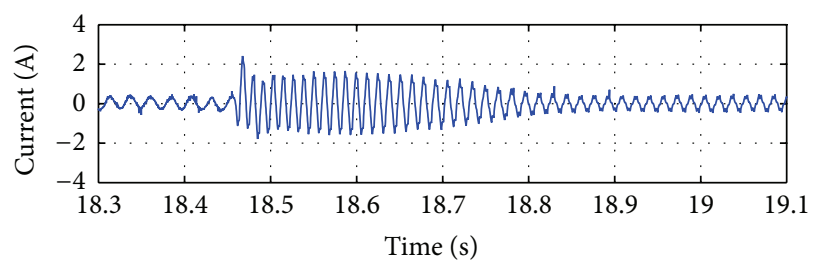

(c)

FIGURE 14: Experimental results for system operation at 1200-1800 rpm. 


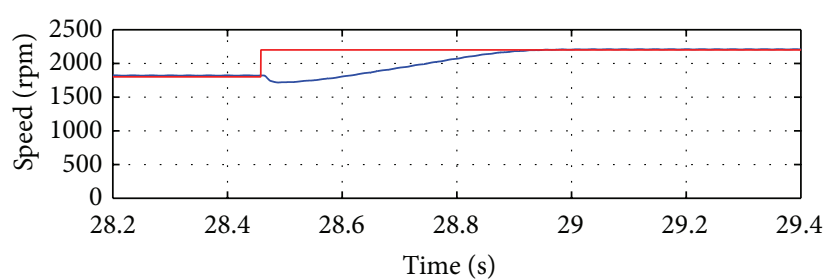

(a)

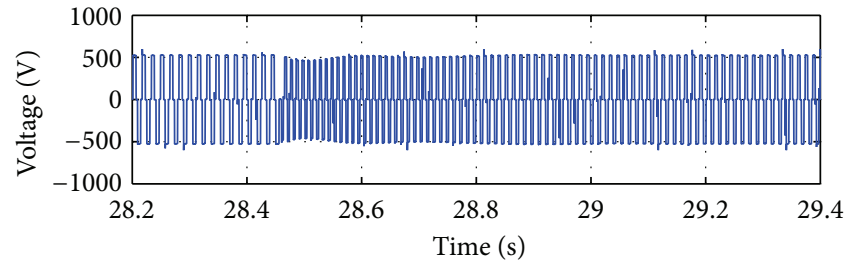

(b)

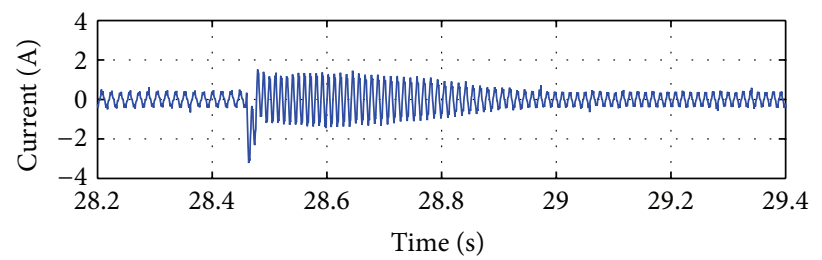

(c)

FIGURE 15: Experimental results for system operation at 1800-2200 rpm.

line current for these operating conditions. The experimental results are enlarged and displayed in detail in Figures 14 and 15. Figure 14 represents the experimental results at 1200 $1800 \mathrm{rpm}$ operation. The operation of the machine at 1800 $2200 \mathrm{rpm}$ is shown in Figure 15. It is evident from Figure 14(b) that the line voltage of the machine changes from sine triangle modulation waveform to that of the square wave modulation. Hence, the system successfully switches between the two modulations techniques without any discontinuity which occurs during the conventional mode switching.

\section{Conclusion}

A variable speed drive system that uses SPWM below base speed operations and square wave technique above the base speed has been successfully simulated and implemented. Based on the simulations and experimentations results, it can be concluded that this hybrid approach combines the advantages of square wave and SPWM for all speed ranges for scalar control of induction machine. The issue of lower order harmonics in square wave operation when operating below base speed is overcome by using SPWM inverter. When operating at the high speed in the field weakening region the square wave operation is more practical because it provides a higher fundamental line-to-line voltage applied to the motor. The transition between the two modes of operation is made seamless by simply setting the frequency of carrier signal to zero. The computer simulations show that the torque and thus the speed response of the proposed algorithm are better than the conventional approach in which inverter mode is simply switched from sine triangle to square wave inverter. The proposed technique is experimentally validated in the lab as well and shows the potential for increasing the efficiency and providing better performance for high speed, high power traction and industrial drives applications.

\section{Nomenclature}

$n_{\text {sync }}:$ Synchronous speed of motor

$P: \quad$ Number of poles

$\omega_{\text {ref }}:$ Reference speed

$\omega_{r}: \quad$ Rotor speed

$\omega_{s}: \quad$ Slip speed

$\omega_{e}: \quad$ Inverter command frequency

$V_{\text {abc }}$ : Inverter output voltage

$V_{\mathrm{abc}}$ : Inverter command voltage

$f^{*}$ : Command frequency to inverter

$V_{\mathrm{dc}}: \quad$ DC bus voltage of inverter

$f: \quad$ Electrical frequency

PI: Proportional integral controller

e: $\quad$ Error signal

$m_{a}$ : Modulation index

$m_{f}:$ Frequency modulation ratio

$f_{c}$ : Carrier frequency

$f_{h}:$ Frequencies at which harmonics occur

$f_{1}$ : Fundamental frequency.

\section{Conflict of Interests}

The authors declare that there is no conflict of interests regarding the publication of this paper.

\section{References}

[1] J. Ewanchuk and J. Salmon, "A Square-wave Controller for a high speed induction motor drive using a three phase floating bridge inverter," in Proceedings of the 2nd IEEE Energy Conversion Congress and Exposition (ECCE '10), pp. 2584-2591, Atlanta, Ga, USA, September 2010.

[2] A. H. Eltom and A. Demirbas, "Motor system energy efficiency in the nylon industry: a comparison of PWM and square wave inverters," in Proceedings of the IEEE International Electric Machines and Drives Conference (IEMDC '09), pp. 508-513, Miami, Fla, USA, May 2009. 
[3] T. Halkosarri and V. Oyi, "Optimal U/f-control of high speed permanent magnet motors," in Proceedings of the IEEE International Symposium on Industrial Electronics (ISIE '06), vol. 3, pp. 2303-2308, Montreal, Canada, July 2006.

[4] Y. Wang, X. Wen, and F. Zhao, "A proposed control strategy of PMSM for deep field-weakening and square-wave mode," in Proceedings of the 15th International Conference on Electrical Machines and Systems (ICEMS '12), pp. 1-6, Sapporo, Japan, October 2012.

[5] W. Song, K. Smedley, and X. Feng, "One-cycle control of induction machine traction drive for high speed railway part II: square wave modulation region," in Proceedings of the 26th Annual IEEE Applied Power Electronics Conference and Exposition (APEC '11), pp. 1003-1009, Fort Worth, Tex, USA, March 2011.

[6] G. Poddar and M. K. Sahu, "Natural harmonic elimination of square-wave inverter for medium-voltage application," IEEE Transactions on Power Electronics, vol. 24, no. 5, pp. 1182-1188, 2009.

[7] H. Cui, F. Wang, Z. Liang, and K. Li, "Current harmonics reduction of square wave inverter by using adjustable filter for high speed motor power supply," in Proceedings of the 11th International Conference on Electrical Machines and Systems (ICEMS '08), pp. 954-957, Wuhan, China, October 2008.

[8] A. Jidin, N. Idris, A. H. M. Yatim, and M. Elbuluk, "A novel overmodulation and field weakening strategy for direct torque control of induction machines," in Proceedings of the IEEE Industry Applications Society Annual Meeting (IAS '08), pp. 1-8, Edmonton, Canada, October 2008.

[9] A. Ghaderi, M. Sugai, T. Umeno, and Y. Amano, "A new direct torque control for AC motors with over modulation ability," in Proceedings of the IEEE International Electric Machines and Drives Conference (IEMDC '11), pp. 1247-1252, Niagara Falls, Canada, May 2011.

[10] M. H. Rashid, Power Electronics Circuits, Devices and Applications, Prentice Hall, New York, NY, USA, 3rd edition, 2004. 

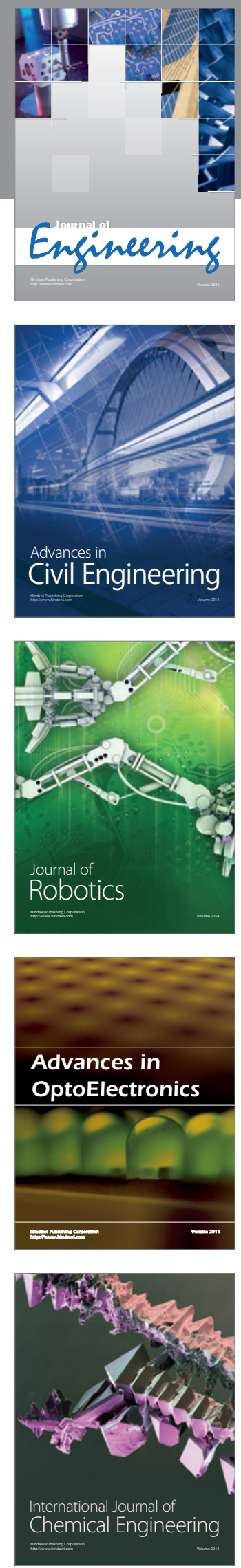

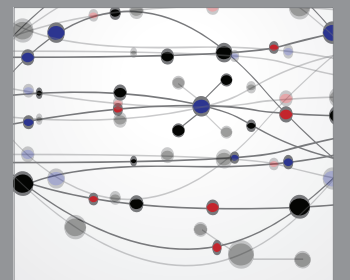

The Scientific World Journal
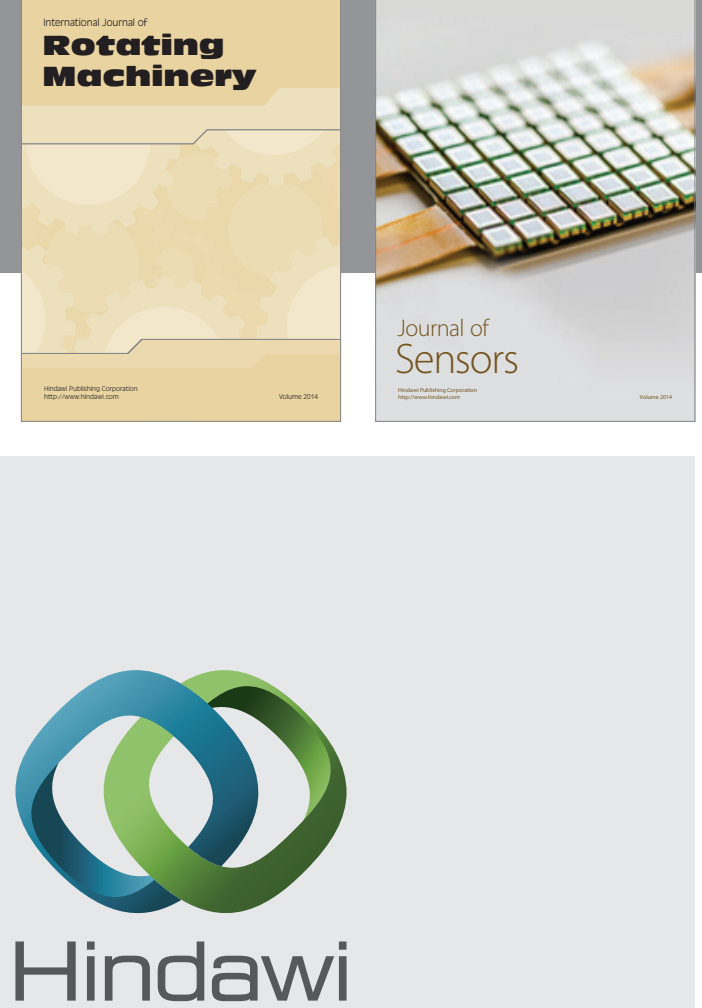

Submit your manuscripts at http://www.hindawi.com
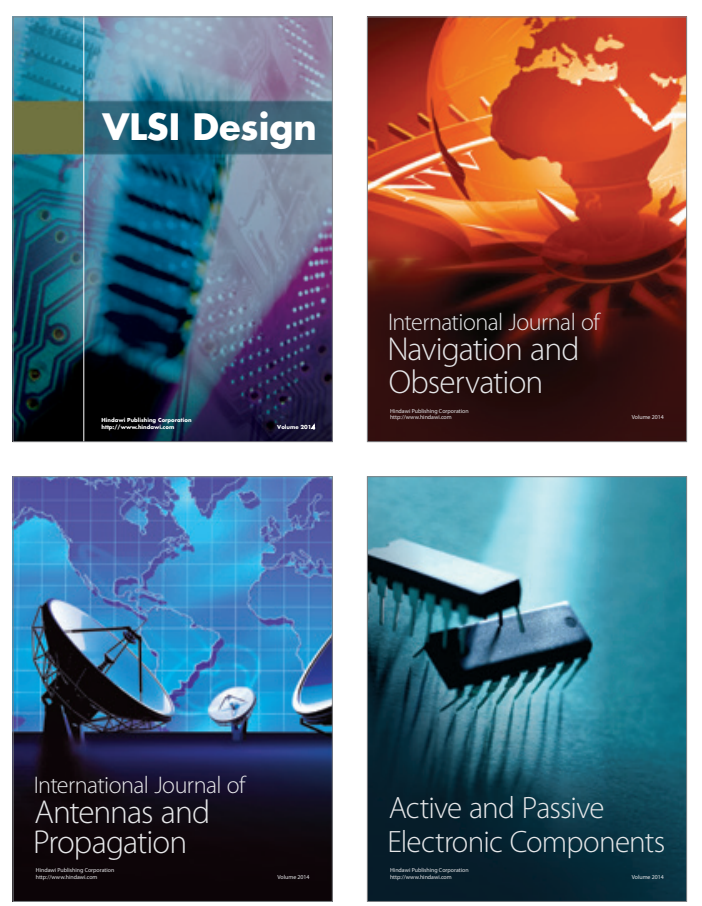
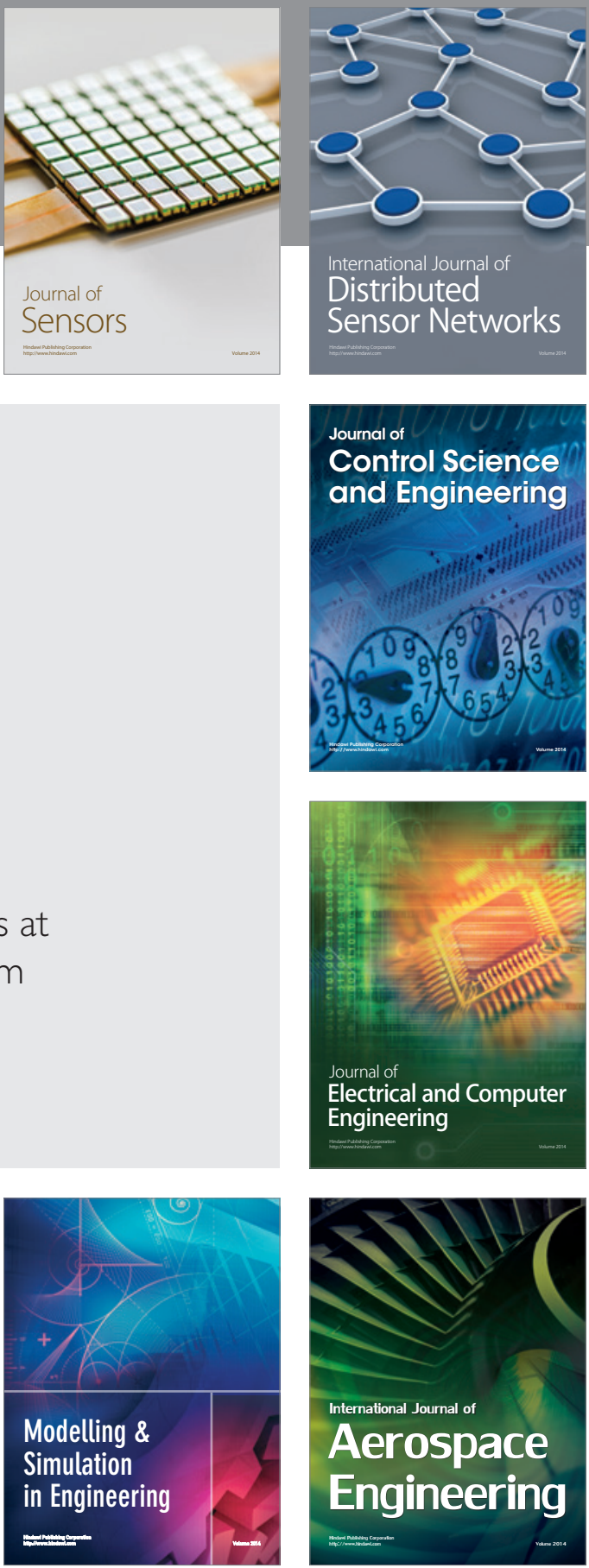

Journal of

Control Science

and Engineering
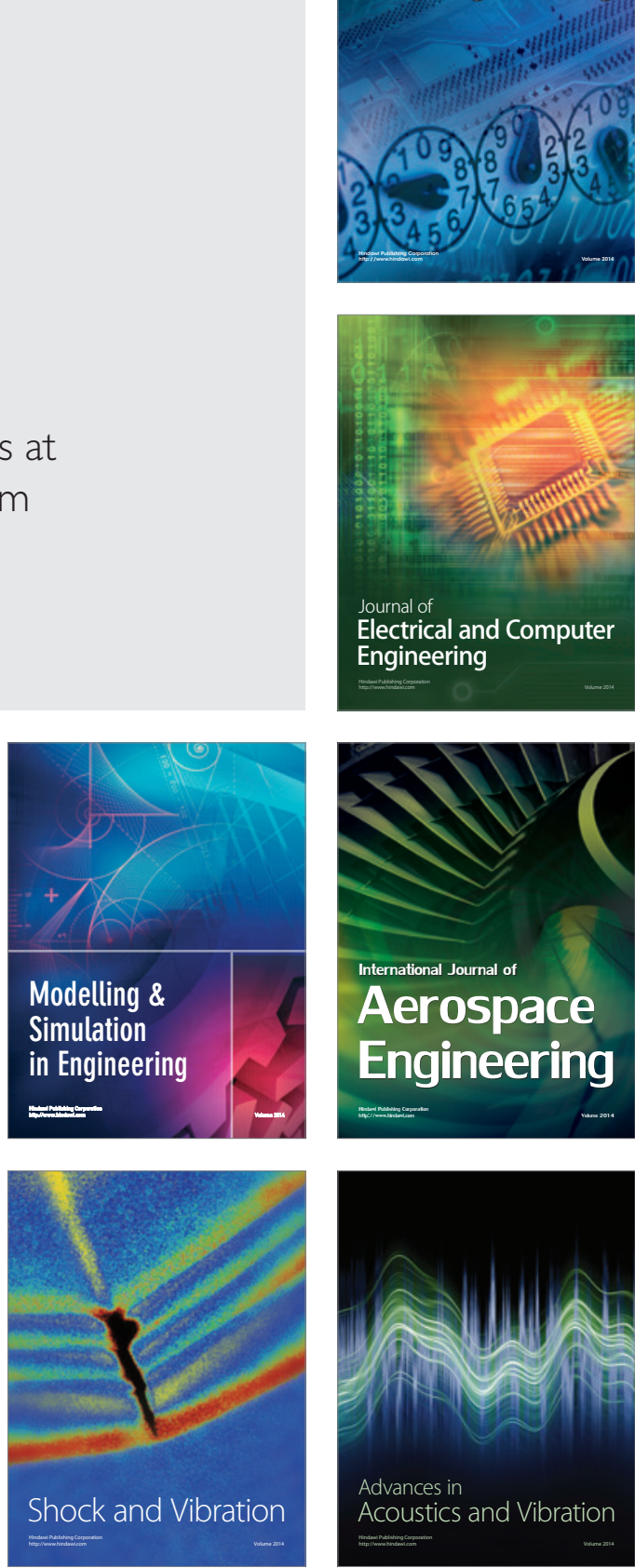